\title{
Alunos em risco na fluência da leitura
}

\section{Students at risk in reading fluency}

\author{
Susana Simões, Ana Paula Loução Martins \\ Instituto de Educação, Universidade do Minho, Centro de Investigação em Educação (CIEd)
}

\begin{abstract}
Resumo
Este estudo teve por finalidade conhecer o impacto da variável risco nas trajetórias do nível da identificação de fonemas, grafemas e fluência leitora desde o final do préescolar ao final do $1 .^{\circ}$ ano do Ensino Básico. Recolhemos dados com provas de monitorização com base no currículo junto de - 22 crianças de um agrupamento Português. Concluímos que para a fluência do som e o nome das letras a diferença entre os alunos em risco e os que não estão em risco diminui ao longo do tempo; enquanto a diferença para os resultados da fluência da leitura aumenta.
\end{abstract}

Palavras-chave: risco na fluência da leitura; monitorização com base no curriculum.

\section{Abstract}

This study aimed to know the level of reading fluency throughout the 1st year of Basic Education and its relation with the level of identification of phonemes and graphemes in preschool. We collected data with monitoring evidence based on the curriculum with - 22 children from a Portuguese grouping. We conclude that the difference between students at risk and those who are not at risk decreases in what concerns the Fluency in the name and sound of the letters average, and increases in what concerns fluency in reading.

Keywords: Risk in reading fluency; Monitoring based on the curriculum.

$\mathrm{Na}$ idade de frequência do pré-escolar saber relacionar a forma de uma letra com o seu nome e com um ou mais dos seus sons é um dos melhores preditores da aquisição da leitura e soletração numa fase mais tardia do percurso escolar (Levin, Shatil-Carmon, \& Asif-Rave, 2006). A pesquisa de Stage, Sheppard, Davidson e Browning (2001) mostrou que os alunos que tiveram um crescimento limitado de fluência leitora no primeiro ano eram aqueles que indicaram apenas oito nomes de letras por minuto no jardim-de-infância. Adicionalmente, mostrou que foi a identificação do nome das letras e não do seu som que mais significativamente contribuiu para o crescimento da fluência leitora no $1^{\circ}$ ano de aprendizagem formal (Stage et al., 2001). Levin e colegas em 2006 desenvolveram um estudo em Israel que teve como objetivo investigar o conhecimento que as crianças tinham sobre o nome das letras e respetivos sons, assim como a sua contribuição para o reconhecimento de palavras. Neste estudo as crianças aprenderam a associar mais facilmente as letras ao som do que ao nome e treinar os sons e os nomes das letras pareceu promover a alfabetização a um nível semelhante, quando esta é medida pelo reconhecimento de palavras. Assim, os investigadores sugerem que professores, pais e investigadores devem usar programas de intervenção que ensinem os sons e nomes das letras concomitantemente, desde tenra idade, para melhorar a alfabetização das crianças (Levin et al., 2006). Em 2011, Dagli realizou um estudo com 2481 alunos do $1^{\circ}$ ano e comparou os resultados daqueles cuja língua Inglesa não era a sua língua materna com os daqueles alunos cuja língua materna era o Inglês. Neste estudo, foram utilizadas as provas de identificação do som e do nome das letras no momento da entrada no jardim-de-infância, no sentido de se perceber qual o papel preditivo destas provas para o 1. ${ }^{\circ}$ Ciclo. Após o estudo, os pesquisadores constataram que a prova de identificação do nome das letras foi a que obteve melhores resultados preditivos para ambos os grupos. Burke, Hagan-Burke, Kwok e Parker (2009) salientam que a investigação tem enfatizado a importância da consciência fonológica, da descodificação fonémica e de uma descodificação automática no desenvolvimento da leitura. Professores do ensino regular e especial devem utilizar indicadores de alfabetização precoce válidos, eficientes e eficazes para triagem e que acompanhem ao longo do percurso escolar, de maneira a prever adequadamente os resultados de leitura. $\mathrm{O}$ desenvolvimento e a validação de indicadores criteriosos de capacidade de alfabetização precoce para crianças, com a finalidade de prevenir a falha da leitura e o seu insucesso deve continuar a ser uma prioridade (Burke et al., 2009). Atualmente, a Monitorização com Base no Currículo (MBC) ${ }^{1}$ é utilizada no contexto do modelo denominado de Resposta-à-intervenção ${ }^{2}$, definido como uma mudança no comportamento, ou no nível de realização académica, em função de uma intervenção específica (Gresham, 2002), como forma de identificar alunos em risco e monitorizar a aprendizagem da leitura. Deno (2003) refere que tem sido desenvolvida muita investigação para mostrar que a MBC pode ser usada para reunir dados de desempenho do estudante e assim ser o suporte de uma série de decisões educacionais. Para Rasinski (2004) o CBM ao permitir a identificação imediata dos alunos que não estão a fazer um progresso adequado e que podem

${ }^{1}$ Tradução de Curriculum based assessment (Deno, 1985)

2 Tradução de Response to intervention. Vários autores utilizam como termos alternativos response-to-instruction e response-to-treatment.

Correspondencia: Susana Simões, susasimoes@ hotmail.com

Selección y peer-review bajo responsabilidad del Comité Organizador del XIV Congreso Internacional Galego-Portugués de Psicopedagogía 
necessitar de medidas adicionais, mais intensivas, ou uma medida mais direcionada, bem como o acompanhamento mais constante de monitorização do progresso para avaliar a eficácia da instrução (Rasinski, 2004). Neste contexto, os alunos considerados em risco são aqueles cujos resultados se situam no ou abaixo do percentil 20 (Deno, 2003).

Neste contexto, realizamos um estudo longitudinal para verificamos o impacto da variável risco na trajetória do nível da Fluência dos sons das letras (FSL), dos nomes das letras (FNL) e da leitura (FL) do pré-escolar ao final do $1 .^{\circ}$ ano de $1^{\circ}$ Ciclo do Ensino Básico.

\section{Método}

\section{Participantes}

A amostra deste estudo é constituída por 22 crianças de idade pré-escolar de um agrupamento do distrito do Norte de Portugal, que posteriormente ingressam em seis escolas do Ensino Básico do 1. ${ }^{\circ}$ Ciclo do mesmo agrupamento. Depois de iniciarmos a análise dos dados, verificamos que existiam três alunos da amostra que consideramos como outliers. Estes alunos não foram retirados do estudo, mas os seus resultados foram analisados à parte. Eram 10 raparigas e 9 rapazes. Os outliers eram dois rapazes e uma rapariga.

\section{Instrumentos}

Foram utilizados três provas de Monitorização com Base no Currículo (Deno, 1985) com o objetivo de recolher dados sobre a Fluência do som das letras, a Fluência do nome das letras e a Fluência da Leitura.

Para a recolha de dados relativos ao som e nome das letras elaboramos uma prova que foi traduzida e adaptada de Fuchs e Fuchs (2007). Esta consiste numa exposição das 26 letras do alfabeto disposta aleatoriamente em maiúsculas, sendo que quatro delas estão repetidas para servirem de exemplo a dar ao aluno antes de a prova ser iniciada. Foi usada uma folha de pontuação e cotação para o som das letras e outra para o nome das letras e seguidas as instruções de administração das provas contidas no guião (Fuchs \& Fuchs, 2007). Estas provas foram administradas individualmente e os alunos tinham que nomear o som das letras ou o nome das letras ao longo de um minuto. A pontuação era atribuída consoante o número de nomes das letras ou som das letras corretos durante esse minuto. A pontuação máxima é de 26 respostas corretas (Fuchs \& Fuchs, 2007), sendo que apenas foi pedido um fonema for letra.

Para a recolha de dados de fluência da leitura, elaboramos uma prova de MBC constituída por três textos (Deno, 1985). Essa preparação foi feita através da seleção de três textos a partir dos manuais escolares de Português do $1 .^{\circ}$ ano, que não estivessem a ser utilizados nas escolas que fizeram parte deste estudo. Os textos tinham entre 32 a 91 palavras. As provas foram administradas individualmente e os alunos tinham que ler os três textos em voz alta durante um minuto. A pontuação de cada prova era o número de palavras lidas corretamente ao longo de um minuto (pcpm) (Deno et al., 2001; Fuchs \& Fuchs 2007). Segundo Deno et al., (2001) a mediana consiste numa estimativa mais precisa e fiável do nível de desempenho dos alunos na leitura, por isso foi este o utilizado para obter o resultado final de cada aluno (mediana das três provas).

\section{Procedimentos}

Todas as provas foram aplicadas em contexto escolar e para iniciar este estudo foi essencial pedir as autorizações necessárias. Os dados foram recolhidos em quatro momentos: no final do pré-escolar (Julho de 2014) foram aplicadas as provas de identificação do nome das letras e de identificação do som das letras. No $1 .^{\circ}$ ano de escolaridade (Dezembro de 2014, Março e Junho de 2015) foram aplicadas as provas de identificação do nome das letras, de identificação do som das letras e Fluência da leitura (tendo em conta que a leitura é iniciada apenas no $1 .^{\circ}$ ano de escolaridade).

No sentido de se garantir a fiabilidade da administração das provas, foram seguidos sempre os mesmos procedimentos e lido sempre no início de cada aplicação o mesmo guião que foi criado previamente com as instruções para o aluno e investigador. Em todas as provas foi utilizado um cronómetro para contabilizar o tempo.

Para descrever os dados recolhidos foram utilizadas medidas de tendência central e de dispersão. Com o objetivo de verificarmos se há diferenças entre momentos temporais, foram realizados testes de Friedman e testes de Wilcoxon com correção de Bonferroni (Field, 2009). Para três momentos temporais foi considerado um nível de significância de .017 $(p=.05 / 3)$ e para quatro momentos $.008(p=.05 / 6)$. Calculou-se o tamanho do efeito para cada um dos testes de Wilcoxon (Field, 2009), utilizando a fórmula proposta por Rosenthal (1991, p. 19, citado por Field, 2009): $r=\mathrm{Z} / \sqrt{ } N$. O valor de $\mathrm{Z}$ corresponde ao z-score indicado no output do SPSS e $N$ corresponde ao número total de observações (Field, 2009). Para verificar a fiabilidade dos resultados foi utilizado neste estudo o método da consistência interna dos itens através da utilização do Alfa de Cronbach.

\section{Resultados}

\section{A trajetória do nível de identificação do nome e do som das letras do final do pré-escolar ao final do $1^{\circ}$ ano de escolaridade}

$\mathrm{Na}$ Tabela 1 apresentamos os resultados dos participantes no estudo, nas provas de MBCidentificação do nome das letras (NL) e identificação do som das letras (SL). A média dos resultados dos alunos na identificação do NL foi superior à do SL na primeira aplicação, o que se inverte nas aplicações seguintes. 
Tabela 1.

Resultados da identificação do som e do nome das letras

\begin{tabular}{lccccc}
\hline Descrição do Item & $\mathrm{N}$ & $\begin{array}{c}\text { Mín } \\
\text {. }\end{array}$ & Máx. & Média & DP \\
\hline NL 1 ${ }^{\text {a }}$ aplicação & 19 & 1 & 21 & 7.6 & 4.8 \\
NL 2 ${ }^{\text {a }}$ aplicação & 19 & 4 & 20 & 12,3 & 5.1 \\
NL 3 ${ }^{\text {a }}$ aplicação & 19 & 5 & 22 & 12.3 & 5.5 \\
NL 4 4 aplicação & 19 & 8 & 26 & 14.3 & 5.3 \\
SL 1 ${ }^{\text {a }}$ aplicação & 19 & 2 & 13 & 6.5 & 3.3 \\
SL 2 2 aplicação & 19 & 5 & 22 & 12.8 & 4.2 \\
SL 3 ${ }^{\text {a }}$ aplicação & 19 & 14 & 22 & 18.9 & 2.2 \\
SL 4 4 aplicação & 19 & 17 & 24 & 20.6 & 1.9 \\
\hline
\end{tabular}

A análise dos quatro momentos da identificação do nome das letras por aluno permite indicar que no último momento de recolha de dados apenas um aluno da amostra apresenta o conhecimento do nome das 26 letras do alfabeto. Nenhum atinge o resultado máximo para o som das letras. Os resultados para a identificação do nome das letras para os alunos que foram considerados outliers mostram que na primeira aplicação, dois destes alunos apresentam o resultado máximo nesta prova, ou seja 26. Na última aplicação estes alunos apresentam resultados de 21 , e 26 nomes das letras corretos por minuto. No que respeita a identificação do som das letras os resultados dos outliers vão aumentando progressivamente ao longo das quatro aplicações. Na quarta aplicação os três alunos apresentam valores muito próximos e elevados de 22 e 23 sons das letras corretos por minuto.

O teste Friedman ANOVA mostrou que existem diferenças estatisticamente significativas entre os quatro momentos de recolha de dados, $\chi^{2}(3)=15,75, p<0.05$.

$\mathrm{O}$ teste de Friedman indica que os resultados da fluência do nome das letras evolui ao longo do tempo de forma estatisticamente significativa, $\chi^{2}(2)=15.75, p=$ .001 Os testes de Wilcoxon com correção de Bonferroni revelam que os resultados da fluência do nome das letras aumenta de forma significativa da primeira aplicação para a segunda, $Z=-2.88, p=.004, r=-66$. O que já não acontece da segunda para a terceira aplicação, $Z=-0.21$, $p=.831$ e da terceira para a quarta aplicação, $Z=-1.51$, $p=.131$. As diferenças da segunda para a quarta aplicação também não são estatisticamente significativas, $\mathrm{Z}=-1.09, p=.275$. As diferenças entre a primeira $\mathrm{e}$ terceira aplicação, $\mathrm{Z}=-2.82, p=.005, r=-65$; assim como da primeira para a quarta aplicação são estatisticamente significativas, $\mathrm{Z}=-3.45, p=.001, r=$ 79. O teste Friedman ANOVA mostrou que existem diferenças entre os quatro momentos de aplicações das provas de fluência dos sons das letras, $\chi^{2}(3)=50,48, p$ $<0.05$. Os resultados para o percentil 20 (ver Tabela 2 ) representam a trajetória dos alunos de risco.
Tabela 2.

Comparação dos resultados por percentil para a identificação do som e do nome das letras

\begin{tabular}{|c|c|c|c|c|c|}
\hline Descrição do Item & $\overline{10}$ & 20 & 50 & 70 & 90 \\
\hline NL $1^{\mathrm{a}}$ aplicação & 3 & 4 & 7 & 10 & 16 \\
\hline NL $2^{a}$ aplicação & 4 & 7 & 12 & 17 & 18 \\
\hline NL $3^{\mathrm{a}}$ aplicação & 7 & 7 & 10 & 15 & 21 \\
\hline $\mathrm{NL} 4^{\mathrm{a}}$ aplicação & 9 & 10 & 13 & 16 & 24 \\
\hline SL $1^{\text {a }}$ aplicação & 2 & 3 & 6 & 7 & 12 \\
\hline SL $2^{\mathrm{a}}$ aplicação & 6 & 9 & 13 & 15 & 19 \\
\hline SL $3^{\mathrm{a}}$ aplicação & 15 & 17 & 19 & 20 & 22 \\
\hline SL $4^{\mathrm{a}}$ aplicação & 17 & 19 & 21 & 22 & 22 \\
\hline
\end{tabular}

A Tabela 3 compara a trajetória do nível de identificação do nome e do som das letras do final do préescolar ao final do $1^{\circ}$ ano de escolaridade dos alunos de risco com aqueles que não estão em risco.

Tabela 3.

Resultados segundo a variável risco para a identificação do som e do nome das letras nas quatro aplicações

\begin{tabular}{|c|c|c|c|c|}
\hline \multirow[t]{2}{*}{$\begin{array}{c}\text { Alunos/ Descrição } \\
\text { do Item }\end{array}$} & \multicolumn{2}{|c|}{$\begin{array}{l}\text { Estão em } \\
\text { risco }\end{array}$} & \multicolumn{2}{|c|}{$\begin{array}{c}\text { Não estão em } \\
\text { risco }\end{array}$} \\
\hline & Média & $\mathrm{DP}$ & Média & $\mathrm{DP}$ \\
\hline NL $1^{\mathrm{a}}$ aplicação & 3 & 1.2 & 9.7 & 4.3 \\
\hline NL $2^{\mathrm{a}}$ aplicação & 5.5 & 1.7 & 14.13 & 4.0 \\
\hline NL $3^{\mathrm{a}}$ aplicação & 6.6 & 0.8 & 14.29 & 4.9 \\
\hline NL $4^{\mathrm{a}}$ aplicação & 9.5 & 0.8 & 16.5 & 4.9 \\
\hline SL $1^{\mathrm{a}}$ aplicação & 5.2 & 4.1 & 6.9 & 3.1 \\
\hline SL $2^{\mathrm{a}}$ aplicação & 7.6 & 1.9 & 14.71 & 2.9 \\
\hline SL $3^{\mathrm{a}}$ aplicação & 16 & 1.4 & 20 & 1.3 \\
\hline SL $4^{\mathrm{a}}$ aplicação & 17.75 & 0.9 & 21.4 & 1.1 \\
\hline
\end{tabular}

Os alunos de risco embora tendo sempre resultados mais baixos que os seus colegas, a diferença entre grupos diminui, sendo que no final do pré-escolar é de 6.7 e no final do $1^{\circ}$ ano do Ensino Básico é de 3.65.

\section{A trajetória do nível de fluência leitora ao longo do primeiro ano de escolaridade}

A média dos resultados dos alunos nos três momentos de recolha de dados (ver Tabela 4) mostram a evolução ao longo do ano letivo.

Tabela 4.

Resultados da população para a fluência da leitura

\begin{tabular}{lccccc}
\hline Momentos & $\mathbf{N}$ & $\begin{array}{c}\text { Mín } \\
\text {. }\end{array}$ & Máx. & Média & DP \\
\hline $\begin{array}{l}1^{\mathrm{a}} \text { aplicação } \\
(12 / 2014)\end{array}$ & 19 & 2 & 6 & 4.42 & 1.12 \\
$\begin{array}{l}2^{\mathrm{a}} \text { aplicação } \\
(03 / 2015)\end{array}$ & 19 & 6 & 31 & 11.47 & 5.82 \\
$\begin{array}{l}3^{\mathrm{a}} \text { aplicação } \\
(06 / 2015)\end{array}$ & 19 & 7 & 42 & 18.89 & 9.66 \\
\hline
\end{tabular}

Uma análise dos resultados das três aplicações da Fluência da Leitura por aluno permite verificar que os 
alunos que apresentam os resultados mais altos de pcpm na segunda aplicação são também os que exibem os valores mais altos na terceira aplicação. No entanto, os alunos que apresentam os valores mais baixos são aqueles que o valor de pcpm menos aumenta ao longo das três aplicações. Dos 19 alunos participantes no estudo nenhum teve resultados iguais ou superiores a $55 \mathrm{pcpm}$ ao longo das três aplicações, sendo que o valor mais alto encontrado é de $42 \mathrm{pcpm}$. Contudo o número de pcpm foi aumentado ao longo das três aplicações.

Os três alunos que apresentam resultados altos de pcpm, logo na primeira aplicação, e por isso considerados outliers, apresentam níveis de fluência da leitura que vão aumentando substancialmente ao longo das três aplicações. Destes três alunos participantes no estudo todos apresentam resultados superiores a $55 \mathrm{pcpm}$ na terceira aplicação, sendo que o valor mais alto encontrado é de 89 pcpm. O número de pcpm foi aumentado ao longo das três aplicações, sendo os únicos do grupo a atingirem as metas curriculares. Sublinhamos que um deles na primeira aplicação já tinha atingido essas metas.

$\mathrm{O}$ teste Friedman ANOVA mostrou que existem diferenças estatísticas significativas entre os resultados da fluência leitora obtidos nos três momentos de recolha de dados com $\chi^{2}(2)=36,11, p<0.05 . p<0.05$.

$\mathrm{O}$ teste de Friedman indica que a fluência da leitura dos alunos participantes evolui ao longo do tempo de forma estatisticamente significativa, $\chi^{2}(2)=36.11, p<.001$

Finalmente, foi feita uma análise ao crescimento semanal do número de palavras corretas por minuto (pcpm), que foi obtido pelo cálculo do coeficiente entre a diferença entre os resultados de duas aplicações e o número de semanas entre as aplicações (Fuchs \& al., 1994; Graney, Missall, Martinez, \& Bergstrom, 2009). Os resultados mostram que da primeira para a segunda aplicação (14 semanas) a taxa de crescimento foi de 0.50 , enquanto da segunda para a terceira aplicação (12 semanas) foi de 0.62. A taxa de crescimento semanal é mais elevada no último terço do ano escolar.

Os resultados para o percentil 20 (ver Tabela 5) representam a trajetória dos alunos de risco na fluência leitora.

Tabela 5.

Comparação dos resultados por percentil para a fluência leitora nas três aplicações

\begin{tabular}{lccccc}
\hline Descrição do Item & $\mathbf{1 0}$ & $\mathbf{2 0}$ & $\mathbf{5 0}$ & $\mathbf{7 0}$ & $\mathbf{9 0}$ \\
\hline $1^{\text {a }}$ aplicação & 3 & 3 & 5 & 5 & 6 \\
$2^{\mathrm{a}}$ aplicação & 6 & 7 & 11 & 13 & 18 \\
$3^{\mathrm{a}}$ aplicação & 7 & 11 & 17 & 25 & 31 \\
\hline
\end{tabular}

Da amostra total verificamos que os alunos em risco não evoluem tanto quanto os alunos que não estão em risco. Passamos agora a mostrar a diferença entre os alunos de risco e não risco na média dos resultados ao longo do $1^{\circ}$ ano do Ensino Básico (ver Tabela 6).
Tabela 6.

Comparação dos resultados segundo a variável risco para a fluência da leitura

\begin{tabular}{ccccc}
\hline Alunos & \multicolumn{2}{c}{ Estão em risco } & \multicolumn{2}{c}{ Não estão em risco } \\
\cline { 2 - 5 } /Momento & Média & DP & Média & DP \\
$1^{\text {a }}$ Aplicação & 3.44 & 0.73 & 5.30 & 0.48 \\
$2^{\text {a }}$ Aplicação & 6.5 & 0.5 & 12.8 & 5.9 \\
$3^{\text {a }}$ Aplicação & 9.00 & 2.0 & 22.43 & 8.76 \\
\hline Nota: $N=19$ & & & &
\end{tabular}

A taxa de crescimento para a fluência da leitura nos alunos em risco (0.213) é três vezes menor do que a taxa dos alunos que não estão em risco (0.658).

\section{Fiabilidade dos resultados}

O valor de Alfa de Cronbach da prova da MBC Fluência oral da leitura, para a primeira aplicação foi de 0.647, para a segunda aplicação foi de 0.946 e para a terceira aplicação foi de 0.952, e mostram que a monitorização com base no currículo se torna mais fiável à medida que o ano letivo. Os resultados para a identificação do Nome das Letras por aplicação têm alfas Alfa de Cronbach entre de 0.845 , de 0.831 , de 0.872 e de 0.867 , respetivamente ao longo dos quatros momentos de aplicação. Os resultados do Alfa de Cronbach para a identificação do Som das Letras são mais baixos e variam entre $0.727,0.814,0.523$ e 0.515 entre o pré-escolar e o final do $1^{\circ}$ ano de escolaridade. Estes valores atestam a boa consistência interna do instrumento uma vez que este valor é superior ao valor mínimo de referência de 0.70 (Almeida \& Freire, 2000).

\section{Discussão}

No $1 .^{\circ}$ ano de escolaridade os descritores de desempenho para a disciplina de Português, nomeadamente para o domínio do conteúdo da leitura e escrita, para o objetivo de conhecer o alfabeto e os grafemas, está previsto nomear a totalidade das letras do alfabeto e pronunciar os respetivos segmentos fónicos (Buescu \& al, 2015). Para a Fluência do nome das letras em média, a prestação dos alunos duplicou da primeira aplicação para a quarta. Em relação à Fluência do som das letras, em média a prestação dos alunos triplicou o seu crescimento da primeira aplicação para a quarta. Concluímos que só um aluno da amostra apresenta o conhecimento do nome das 26 letras do alfabeto e nenhum atinge o resultado máximo para o som das letras; os resultados mostram uma evolução ao longo do tempo do nível de conhecimento do som e do nome das letras.

$\mathrm{Na}$ Fluência da leitura em média, a prestação dos alunos na primeira aplicação foi de $4.42(D P=1.121)$ pcpm, já na terceira aplicação o valor foi de 18.89 $(D P=9.655)$ pcpm, sendo que o valor aumentou quatro vezes da primeira aplicação para a terceira. Assim, concluímos que em média, os alunos não atingiram o nível de fluência de leitura proposto nas Metas de aprendizagem curriculares Portuguesas, embora tenham mostrado evolução significativa na aprendizagem da fluência de leitura. Em média, os alunos ficaram bastante aquém de atingir o nível de referência proposto por Fuchs e Fuchs (2007), que são as 50 pcpm para o $1 .^{\circ}$ ano nos 
EUA, assim como, das 55 pcpm referidas por Buescu et al. (2015) nas metas de aprendizagem curriculares portuguesas.

A taxa de crescimento da fluência da primeira aplicação para a segunda (14 semanas) foi de 0.503 pcpm, da segunda aplicação para a terceira aplicação (12 semanas) foi de 0.618 pcpm, o que nos leva a concluir que o crescimento semanal não chega a uma pcpm por semana. Segundo Deno et al. (2001) o crescimento semanal de 0.49 pcpm é um crescimento modesto o que vem atestar que o crescimento semanal neste estudo é baixo.

Segundo Deno (2003) os alunos que se posicionam abaixo do percentil 20 (inclusive) no que concerne aos resultados obtidos na MBC- fluência da leitura estão em risco de apresentar DAE na leitura (Deno, 2003). Assim, relativamente à variável risco na Fluência da Leitura, concluímos que a diferença entre o grupo de alunos em risco e o de alunos que não são considerados em risco aumenta ao longo do ano, passando essa diferença de 5.56 para 17.13 palavras lidas corretamente por minuto.

No que concerne à taxa de crescimento para a fluência da leitura nos alunos em risco, concluímos que esta é três vezes menor do que a taxa dos alunos que não estão em risco, apresentando um valor de 0.213. Para diminuir este desfasamento era importante que os alunos em risco recebessem intervenções de nível II do modelo de resposta-à-intervenção. Intervenções intensivas num pequeno grupo e baseadas na investigação, como a leitura oral monitorizada e repetida (Chard et al., 2002; NICHD, 2000).

Com os resultados deste estudo concluímos que existem correlações com associações positivas estatisticamente significativas entre resultados, principalmente entre a Fluência do Nome das Letras e a Fluência da Leitura. Existe uma associação positiva estatisticamente significativa entre o resultado da fluência do nome das letras na primeira aplicação e a fluência da leitura na segunda aplicação, $r=.61, p=.005$ e terceira aplicação, $r=.61, p=.005$. Também podemos observar que existe uma associação positiva estatisticamente significativa entre os resultados da fluência do som das letras na segunda aplicação e os resultados da fluência da leitura na primeira aplicação, $r$ $=.61, p=.006$. Para finalizar existe uma associação positiva estatisticamente significativa entre os resultados da fluência do nome das letras na quarta aplicação e fluência da leitura na terceira aplicação, $r=.67, p=.002$.

Neste estudo podemos concluir que a primeira aplicação (pré-escolar) da Fluência do Nome das Letras, explica $37 \%$ dos resultados da Fluência da leitura no final do $1^{\circ}$ ano do Primeiro Ciclo do Ensino Básico, já a fluência do som das letras explica apenas $0.1 \%$ desses mesmos resultados. É também de sublinhar que a Fluência do Nome das Letras no final do $1 .^{\circ}$ ano explica $45 \%$ dos resultados da Fluência da leitura no final do $1 .^{\circ}$ ano do Primeiro Ciclo do Ensino Básico.

$\mathrm{O}$ valor de Alfa de Cronbach em todas as provas utilizadas indicam um valor médio de 0.80 . Estes valores atestam a boa consistência interna do instrumento uma vez que este valor é superior ao valor mínimo de referência (0.70) (Almeida \& Freire, 2000).
Comprovamos mais uma vez a fiabilidade da Monitorização com Base no Currículo, tanto na fluência da leitura como na fluência do som e do nome das letras.

\section{Referências}

Almeida, L. S. \& Freire, T. (2000). Metodologia da investigação em psicologia e educação. Braga: Psiquilíbrios.

Buescu, H. C., Morais, J., Rocha, M. R., \& Magalhães, V. F. (2015). Programa e metas curriculares de Português do ensino básico. Retirado em: 09/07/2015, de http://www.dge.mec.pt/portugues.

Burke, M. D., Hagan-Burke, S., Kwok, O., \& Parker, R. (2009). Predictive validity of early literacy indicators from the middle of kindergarten to second grade. Journal of Special Education, 42 (4), 209-226.

Chard, D. J., Vaughn, S., \& Tyler, B. J. (2002). A synthesis of research on effective interventions for building reading fluency with elementary students with learning disabilities. Journal of Learning Disabilities, 35, 386-406.

Dagli, Y.U. (2011). Predicting ELL students' beginning first grade English oral reading fluency from initial kindergarten vocabulary, letter naming, and phonological awareness skills. Early Childhood Research Quarterly, 26 (1), 15-29.

Deno, S. L. (1985). Curriculum-based measurement: The emerging alternative. Exceptional Children, 52(3), 219-232.

Deno, S L. (2003). Developments in curriculum-based measurement. The Journal of Special Education, 37 (3), 184-192.

Field, A. P. (2009). Discovering statistics using SPSS: and sex and drugs and rock ' $n$ ' roll (third edition). London: Sage publications.

Fuchs, L. S., \& Fuchs, D. (2007). Using CBM for progress monitoring in reading. Students Progress Monitoring. Retirado em 02/08/2015 de http://www.studentprogress.org/library/training/cbm\% 20reading/usingcbmreading.pdf.

Gresham, F. M. (2002). Responsiveness to intervention: An alternative approach to the identification of learning disabilities. In R. Bradley, L. Danielson \& D. P. Hallahan (Eds.), Identification of learning disabilities: Research to practice (pp. 467-519). Mahwah, NJ: Erlbaum.

Levin, I., Shatil-Carmon, S., \& Asif-Rave, O. (2006). Learning of letter names and sounds and their contribution to word recognition. Journal of Experimental Child Psychology, 93(2), 139-165. doi:http://dx.doi.org/10.1016/j.jecp.2005.08.002

NICHD National Institute of Child Health and Human Development. (2000). Report of the national reading panel. Teaching children to read: an evidence-based assessment of the scientific research literature on reading and its implications for reading instruction: Reports of the subgroups (NIH Publication No. 004754). Washington, DC: U.S. Government Printing Office.

National Reading Panel (2000). Report of the National Reading Panel. Teaching children to read: An 
evidence-based assessment of the scientific research literature on reading and its implications for reading instruction: Reports of the subgroups (NIH Publication No. 00-4754). Washington, DC: U.S. Government Printing Office.

Rasinski, T. V. (2004). Assessing reading fluency. Honolulu: Pacific Resources for Education and Learning.

Stage, S. A., Sheppard, J., Davidson, M. M., \& Browning, M. M. (2001). Prediction offirst-Graders' growth in oral reading fluency using kindergarten letter fluency. Journal of School Psychology, 39 (3), 225237. 